\title{
2nd Hypertension Research Award for authors of outstanding papers in HR
}

\author{
Hypertension Research (2012) 35, 131; doi:10.1038/hr.2011.215
}

$\mathrm{J}$

apanese Society for Hypertension (JSH) has announced the winners of the 2nd Hypertension Research Award. This award was established in 2010 to recognize significant contributions of researchers to the advancement of researches in hypertension and related studies. Among the first or main authors of the articles published in Hypertension Research, official journal of JSH, the journal's editorial committee members selected following winners.

\section{HYPERTENSION RESEARCH AWARD OF EXCELLENCE}

Dr Yoshihiro Kokubo, Department of Preventive Cardiology, National Cerebral and Cardiovascular Center, Osaka, Japan.

For contribution of 'The combined impact of blood pressure category and glucose abnormality on the incidence of cardiovascular diseases in a Japanese urban cohort: the Suita Study' Vol. 33, No. 12, pp 1238-1243.

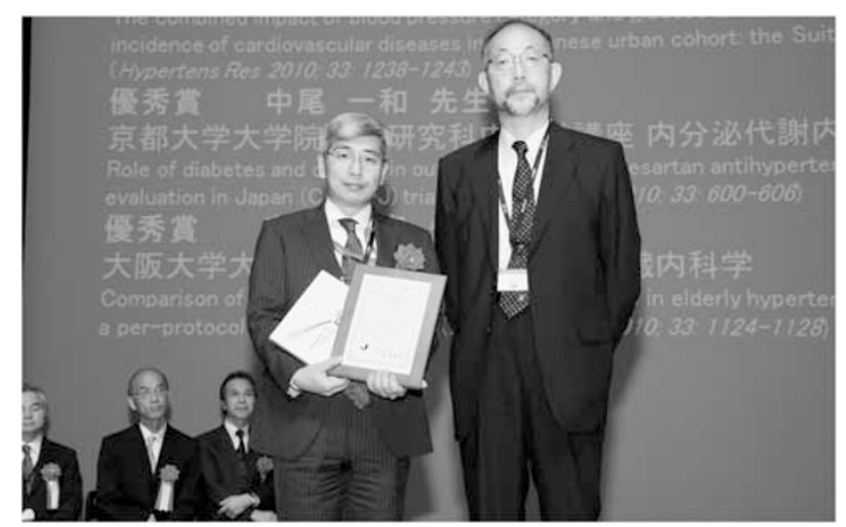

Dr Yoshihiro Kokubo

\section{HYPERTENSION RESEARCH AWARD}

Dr Kazuwa Nakao, Department of Medicine and Clinical Science, Kyoto University Graduate School of Medicine, Kyoto, Japan; EBM Research Center, Kyoto University Graduate School of Medicine, Kyoto, Japan.

For contribution of 'Role of diabetes and obesity in outcomes of the candesartan antihypertensive survival evaluation in Japan (CASE-J) trial', Vol. 33, No. 6, pp 600-606.

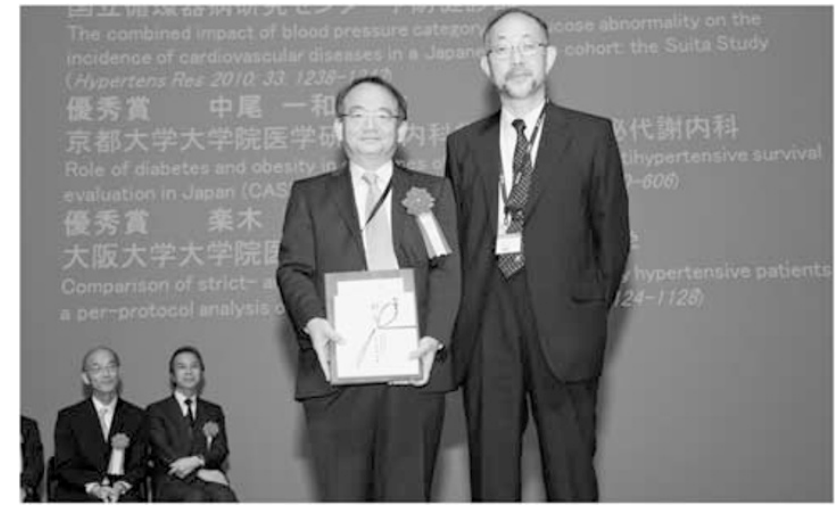

Dr Kazuwa Nakao

\section{HYPERTENSION RESEARCH AWARD}

Dr Hiromi Rakugi, Department of Geriatric Medicine and Nephrology, Osaka University Graduate School of Medicine, Osaka, Japan.

For contribution of 'Comparison of strict- and mild-blood pressure control in elderly hypertensive patients: a per-protocol analysis of JATOS', Vol. 33, No. 11, pp 1124-1128.

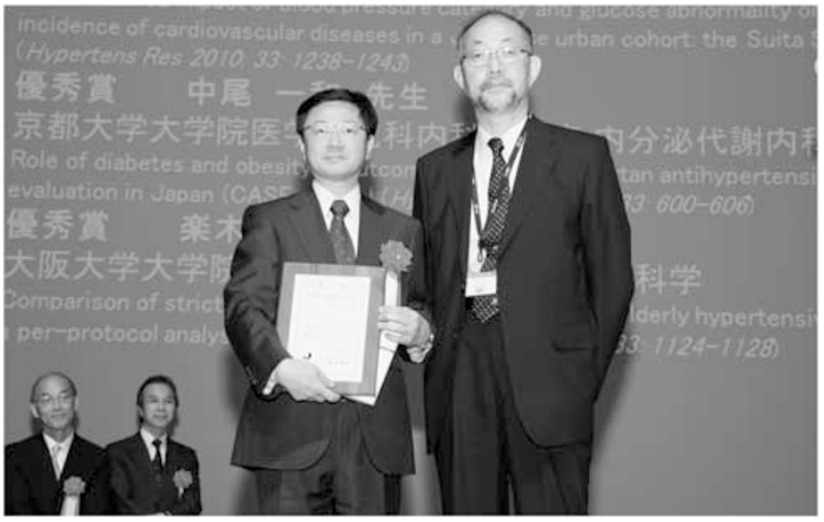

Dr Hiromi Rakugi

Above winners were invited to receive the award at JSH annual meeting on the 21st October in Utsunomiya. Award certification and supplementary were presented from the President of JSH, Professor Kazuyuki Shimada.

Masatsugu Horiuchi Editor-in-Chief Hypertension Research 